\title{
The Pioneer Effect: A New Physics with a New Principle
}

\author{
Russell Bagdoo \\ Saint-Bruno-de-Montarville, Quebec, Canada \\ Email: rbagdoo@gmail.com, rbagdoo@yahoo.ca
}

How to cite this paper: Bagdoo, R. (2020) The Pioneer Effect: A New Physics with a New Principle. Journal of Modern Physics, 11, 616-647.

https://doi.org/10.4236/jmp.2020.115041

Received: March 20, 2020

Accepted: May 5, 2020

Published: May 8, 2020

Copyright (c) 2020 by author(s) and Scientific Research Publishing Inc. This work is licensed under the Creative Commons Attribution International License (CC BY 4.0).

http://creativecommons.org/licenses/by/4.0/

\begin{abstract}
Radiometric data from the Pioneer 10/11, Galileo and Ulysses spacecraft indicated an anomalous constant acceleration acting on them, directed toward the Sun, and a gradual growth of the radio signal frequency emitted by the receding transmitter. The reported odd acceleration of Pioneer 10 with a magnitude $\sim 8.5 \times 10^{-10} \mathrm{~m} / \mathrm{s}^{2}$ can be explained by an induced gravitational interaction on the S-band signals traveling between the probe and the Earth, arising from the electromagnetic properties of the outer Solar System vacuum zero-point radiation interacting with matter. Their nature is of quantum vacuum origin, and these induced forces act in addition to ordinary gravitational forces, violating the principle of Equivalence. We suggest a new physical theory based on a new principle called "Compensation" as a thinkable explanation for the non-conventional Pioneer effect. The theory of Relation, which is an alternative to the inflationist model, postulates that our universe is made of two antagonistic but complementary structures. The principle of Compensation contradicts Relativity theory, predicts such acceleration and is for the electromagnetic spacetime metric what the principle of Equivalence is for the gravific spacetime metric.
\end{abstract}

\section{Keywords}

Theory of Relation, Principle of Compensation, Two Structures, Negative EM Wavelength of Spacetime, Zero Point Fluctuations

\section{Introduction}

First published in 1998 (PRL 81, 2858), results from an almost twenty-year study of radiometric data from Pioneer 10/11, Galileo and Ulysses spacecraft have been continuously reported by Anderson et al. $(1998,1999,2002)$ [1] [2] [3]. Detailed analyses of these data indicated existence of an apparent anomalous, 
constant, acceleration acting on the spacecraft, directed toward the Sun [4]. The spacecraft ranging and Doppler velocity measurements are performed by sending a radio signal from the Earth to the spacecraft, from which an active transponder returns it back. The time of the signal travel is used for the position calculation verification and the Doppler effect of frequency change with its high accuracy provides an additional verification of the relative velocity. Considering $v_{\text {model }}$ [5], the predicted frequency of the re-transmitted signal observed by a DSN antennae, and $v_{o}$ the reference frequency. The observed, two-way normal effect can be expressed to first order in $v / c$ as

$$
v_{\text {model }}=v_{o}\left[1-2 v_{\text {model }}(t) / c\right] \text {. }
$$

The factor 2 is because they use two- and three-way data. $v_{\text {model }}$ is the modeled velocity of the spacecraft due to the gravitational and other large forces. This velocity is outwards and hence produces a red shift. The measurements with the use of special programs permitted to observe a steady increment of frequency of the radio signal sent off from the Earth and actively re-transmitted by the spacecraft, i.e. on the background of the usual Doppler effect, the drift of the measured frequency toward its growth displayed itself and allowed to conclude that Pioneer 10 slows down. Therefore, the acceleration $a_{p}$ produces a slight blue shift on top of the larger red shift

$$
\left[v_{\text {obs }}(t)-v_{\text {model }}(t)\right]_{D S N}=-v_{o}\left(2 a_{p} t\right) / c .
$$

$v_{o b s}$ is the frequency of the re-transmitted signal observed by a DSN antennae. Anderson et al. concluded that there was an anomalous, weak, long-range acceleration of the Pioneer spacecraft in the outer regions of the solar system toward the Sun of $\sim-8.5 \times 10^{-10} \mathrm{~m} / \mathrm{s}^{2}$. The sign indicates that $a_{p}$ is inward [5]. It shows a steady frequency drift of about $-6 \times 10^{-9} \mathrm{~Hz} / \mathrm{s}$, or $1.5 \mathrm{~Hz}$, over 8 years (one-way only). This equates to a clock acceleration, $-a_{t}$, of $-2.8 \times 10^{-18} \mathrm{~s} / \mathrm{s}^{2}$. The identity with the apparent Pioneer acceleration is

$$
-a_{t}=a_{p} / c=-8.5 \times 10^{-10} \mathrm{~m} / \mathrm{s}^{2} / c=-2.88 \times 10^{-18} \mathrm{~s} / \mathrm{s}^{2} .
$$

The S-band carrier radio signal frequency is $v_{o} \approx 2.29 \mathrm{GHz}$

$$
\begin{gathered}
-a_{t} v=v /-t_{\text {cosm }}=-a_{p} v / c \\
\left(-2.88 \times 10^{-18} \mathrm{~s} / \mathrm{s}^{2}\right) \times\left(2.29 \times 10^{9} \mathrm{~Hz}\right) \\
=\left(2.29 \times 10^{9} \mathrm{~Hz}\right) /\left(-3.472 \times 10^{17} \mathrm{~s}\right) \\
=\left(-8.65 \times 10^{-10} \mathrm{~m} / \mathrm{s}^{2}\right)\left(2.29 \times 10^{9} \mathrm{~Hz}\right) / \mathrm{c} \\
=-6.6 \times 10^{-9} \mathrm{~Hz} / \mathrm{s}
\end{gathered}
$$

Besides, an independent analysis by Markwardt [6] of radio Doppler tracking data from the Pioneer 10 spacecraft for the time period 1987-1994 confirms the previous observations. The anomalous accelerations observed are constant and persistent. None of the claimed conventional effects can account for the magnitude and direction of the anomaly. 
We think that the reported anomalous acceleration of the Pioneer 10 spacecraft of $\sim-8.5 \times 10^{-10} \mathrm{~m} / \mathrm{s}^{2}$ (i.e. toward the Sun) and the gradual growth of the radio signal frequency emitted by the receding transmitter can be explained by a gravitational interaction on the S-band signals traveling between Pioneer 10 and the Earth, and by the properties of the interplanetary vacuum.

But before coming to this explanation, let's relate what happened around 2011-2012. Until 2011, after many theories had been advanced, there was no satisfactory explanation for this acceleration anomaly affecting the Pioneer 10 and 11 space probes during their transit outside the solar system and measured between 1979 and 2002. An Italian-Portuguese team in 2011 verifies and validates a relatively prosaic explanation of the Pioneer anomaly. The heat produced by radioisotope thermoelectric generators is emitted isotropically (in all directions), but a significant fraction of it is reflected by the back of the large gain satellite dish. The resulting radiation pressure pushes in the opposite direction, i.e. in the direction of the Sun. These results have been confirmed by independent teams using thermal modeling software and in 2012 the scientific community believes that the mystery is solved.

We believe that blind confidence in this assumption alone of thermal recoil pressure has made these digital models too schematic. In this respect, we consider it useful to submit here another hypothesis which leads to the opposite conclusion: the thrust is in the direction of displacement of the probe.

First, let's mention the main internally generated heat source comes from the spacecraft electronics box located on the leading side of the spacecraft, but just behind the large parabolic high-gain antenna that points to Earth. The box collects the heat of the various scientific instruments (including the heat of the sun captured by the antenna). Orifices of the box facing the top of the antenna curve evacuate the heat. We assume that the arrival of this heat forms a kind of exhaust gas does not crush on the top of the antenna but dissipates in jets on both sides of the axis of the parabola. These jets slide along the opposite side walls and accompany the curves towards the flared end of the parabolic antenna where they are thrown into the void facing the sun. This is how the probe would be pushed forward, in the direction of travel of the spacecraft, by a force called thrust; this under Newton's third law of motion: each action causes an equal reaction and of opposite direction.

The other source of heat comes from two radio-thermal generators (RTGs), cylindrical in shape, each connected to the main compartment through a truss. These nuclear power sources also emit heat toward the back side of the disc-shaped antenna pointing in the direction of the Earth. When the thermal radiation reaches the main compartment of the equipment, where the majority of the energy is consumed, it joins the gas streams evacuated from the electronic box which travel at high speed to the ends of the horn of the antenna to be expelled and generate the forward thrust at the closed interior apex of the antenna.

We conclude that an additional anomaly with a conventional explanation can 
add to the Pioneer effect. And that at the current level of our knowledge of the Pioneer 10 spacecraft and its trajectory, the statistically significant acceleration anomaly still exists and cannot be resolved in standard physics. Its origin must be found in new principles. An unconventional explanation will have astronomical consequences: cosmology, starting from its foundation [7], will have to consider a paradigm shift; standard physics will undergo major renovations, and it will be an opportunity for discovering a new vision of the universe.

In this paper we study the consistency of the data of the Pioneer anomaly with the new theory of Relation. Firstly, in Sec. 2, we introduce the initial conditions of the theory and its new principle. In Part 1 Sec. 3, we show how Pioneer effect is a violation of the equivalence principle. In Sec. 3.1, we postulate the principle of Compensation, which explains the Pioneer anomaly of the outwards redshift in relation with the inward blueshift, and find the deep meaning of this principle in the existence of two structures going in opposite directions. In Sec. 3.2, we suggest that the loss of energy of the photon in the farther interplanetary vacuum induces a gravitational effect like a macroscopic Casimir effect. In 3.3, we understand the vacuum by a possible effect from unknown physics: a gravitational frequency shift of the radio signals proportional to the distance to the spacecraft and a loss of density energy in the intermediate medium. We argue that the gravitational interaction of the S-band radio with a growing empty vacuum might be responsible of the anomalous acceleration of the Pioneer spacecraft. In 3.4, according to the $\mathrm{CP}$, we construe bond between the gravitational photon of the structure of condensation and the cosmological photon of the structure of expansion: energy lost by the latter is retrieved by the former. We discern, in Sec.3.5, gravific spacetime and EM spacetime, and explain in 3.6 the relation between the acceleration of Pioneer inward and its deceleration outwards. Predictions in Sec. 3.7. In Part 11 Sec. 4, the unaccepted concept of "tired light" seems consistent with data of the anomaly and is integrated within the framework of the theory of Relation which claims, in 4.1, that gravitation and electromagnetism are two sides of one force. In Sec. 4.2, with the intention of dispelling a huge discrepancy and to be reconciled with anomalous data, we propose a negative cosmological constant with a varying density of energy through time, which implies a positive pressure. In Sec. 4.3, we discuss of links between CP, zero point fluctuations, induction of gravity and inertia. Along a different line in 4.4, we concentrate on connections between theory of Relation and quantum gravity through Pioneer effect. With correlations and assumptions concerning the graviton, we write down some characterizations of a quantum gravity to be constructed, finally, the conclusion in Sec. 5 .

\section{Relativity, Theory of Relation and Principle of Compensation}

Special Relativity (SR) concerns isolated system, free of outside influence, in a completely empty and homogeneous space in which the speed of light is an ab- 
solute constant. General Relativity (GR) is SR looking at the influence of gravitation. It is based on the principle of Equivalence (EP) considering the equality everywhere between gravitational mass and inertial mass. Matter, which is gravity, curves spacetime.

The new theory called "Theory of Relation" regards that not only matter but also vacuum interact with the system. Vacuum is not empty space but ocean of interacting negative and positive energies. The reported anomalous acceleration of the Pioneer 10 spacecraft is a consequence of this misunderstood part of our physics. This theory gives an interaction between the electromagnetic (EM) spacetime of expansion and the gravitational matter of condensation. This new cosmological model initially assumed that

1) Our universe is made of two complementary and interpenetrated structures, one for condensation with a gravific spacetime and EM matter (Einstein), the other for expansion with a flat EM spacetime and ordinary matter (Lorentz-Maxwell).

2) Since the big bang, the EM structure of expansion is decreasing giving up its energy to the positive gravitational increasing structure of condensation. A perpetual annihilation of the negative energy-mass is transformed into a continual creation of positive energy-mass. The first structure of condensation represents the positive solution of Dirac's equation of energy while the second structure of expansion express its negative energy solution which was eliminated by a mathematical trick [8].

3) The EM wave of spacetime is supported by an inhomogeneous vacuum filled of "minimal" negative energy perpetually in interaction with positive matter. The speed of light is a constant but there is an anisotropy making it not absolute.

4) Ether exists. There is no precise definition for it and it is a lumber room for scientists. In our theory, it is close to the Lorentz's ether theory: a negative EM spacetime wave in a quantum void, which is a plenum.

5) Electromagnetism in macrocosm is the negative side of gravitation [8] [9]. If gravitation is the only force between planets in the structure of condensation, electromagnetism is the driving force of the structure of expansion. Gravitation of the first structure is "positive" relatively to the "negative" electromagnetism of the second structure.

6) The theory is in a framework of "cyclic universe theory". Our model combines the cosmology of the big bang with the continual creation of matter. Our big bang starts with the big crunch of a pre-big bang universe. This pre-compression resolves the problems of causality, conservation, flatness, horizon, without being committed to the cosmological standard model of inflation, strings, higher dimensions, or to branes and orbifolds. The concepts that the universe "comes back full of negative energy-mass", and the "normalization" of the negative energy solution of the classic equations, are the central ideas of this cyclic model.

Our complex universe is dual: positive and negative. The negative part of the 
universe, which is a universe by itself, is disintegrating, "creating" our actual positive universe. The compensation principle $(\mathrm{CP})$ asserts that the permanent loss of negative energy of the expanding EM wavelength of spacetime induces the positive gravific spacetime matter. Flat EM spacetime can yield induced gravity to ordinary matter. Gravific spacetime matter produced by the expansion can flatten the EM spacetime.

Note on matter and antimatter: In the theory of Relation, our dual universe contains two opposite structures of energy-mass, each has two roots for both positive and negative energy, each containing its own particles and antiparticles, being dependant of Dirac's equation on energy

$$
\pm E=\left(m^{2} c^{4}+p^{2} c^{2}\right)^{1 / 2} .
$$

In each structure, antimatter is matter that is composed of the antiparticles of those that constitute normal matter. In a larger sense, antimatter is the extension of the structure of expansion, while our normal matter constitutes almost entirely the structure of condensation. This asymmetry of matter and antimatter would come from a kind of continual transmutation attributed to the disintegration, all along the cosmic expansion, of the negative antimatter-anti energy into the creation of positive matter-energy of the observable universe.

\section{PART 1}

\section{TWO STRUCTURES AND TWO PRINCIPLES}

\section{Violation of the Principle of Equivalence in Empty Inertial Space}

In empty space of GR, a supposed observer inside the Pioneer spacecraft will theoretically undergo a force equal to "inertial mass $\times$ acceleration", also equivalent to a force equal to "gravitational mass $\times$ intensity of the gravitational field" [10]

$$
F_{i}=M_{i} \times a=F_{g}=M_{g} \times g .
$$

This equivalence is corroborated by experiences on Earth. But observation on Pioneer gives

$$
\begin{gathered}
\left(F_{i}=M_{i} \times a\right) \neq\left(F_{g}=M_{g} \times g\right) . \\
\text { Precisely }\left(F_{i}=M_{i} \times a\right)<\left(F_{g}=M_{g} \times g\right) .
\end{gathered}
$$

Keeping the equality between the masses, a decreases while $g$ increases. Anomalous deceleration outwards is interpreted like anomalous acceleration toward the Sun [5]

$$
\Delta g=-a_{t} c=a_{p}=-8.5 \times 10^{-10} .
$$

This anomalous acceleration, which signifies an extra measurable gravitational potential, counteracts the EP saying "In a freely falling local frame (even including self-gravitating bodies), no influence is measurable of the gravitational potential which may cause an acceleration of the frame as a whole" [11]. 
Radio-signal interval is shorter than expected meaning linear radius (Newtonian) is shorter (range). With GR, shorter distance wants to say a greater curvature due to an excess of sunward gravity [12] [13]. Let us suppose a clock situated on a probe in a fieldless region of space. With GR, if we take the origin of the space coordinates in the center of an atom, we can put $\xi(x)=\eta(y)=\zeta(z)=0$, and have

$$
\begin{gathered}
s^{2}=-c^{2} T^{2}=g_{44} t^{2} \\
t=T c /\left(-g_{44}\right)^{1 / 2} .
\end{gathered}
$$

It is only in a fieldless space that we have $g_{44}=-c^{2}$, thus $t=T$.

Suppose a clock situated on Pioneer 10 in the same "fieldless" region of space. An anomalous acceleration is observed: we say that a downward trend of minimal energy with distance in the vacuum induces gravity. So the gravitational field $g_{44}$ in this false fieldless is different from $-c^{2}$, say $g_{44}=-c^{2}(1-\gamma)$. Thus the time of vibration is altered to

$$
t=T /(1-\gamma)^{1 / 2}
$$

or, as the deviation $\gamma$ is small, approximately

$$
t=T /(1+\gamma / 2) ;\left[\gamma=2 \Phi / c^{2}=2 G M / R c^{2}=2 v^{2} / c^{2}\right] .
$$

For GR and the EP, a clock in the vacuum undergoes no gravity. The CP says there is a difference in the beating of the same clock for which the difference of the induced gravitational field in the vacuum given by $g_{44}$ has the relative value $\gamma$.

Pioneer effect is a clear violation of EP, an anomaly in the redshift of GR. It is as if the loss of density in the vacuum was turned into gravitational energy, like if a negative energy was converted into a positive energy. Energy of both systems is conserved if what constitutes a non-standard channel of energy-loss by a is recuperated by $g$. A new principle is necessary to explain this anomalous effect and maintain this conservation.

\subsection{Principle of Compensation in Empty EM Space}

The principle of compensation says that the decrement of negative EM energy-mass during the expansion induces a proportional and opposite increment of the positive gravitational energy-mass. Considering that further distance from matter lowered minimal density energy in vacuum, assuming that a long-range negative scalar field interacts with matter inducing gravity, the $\mathrm{CP}$ permits the transformation of negative EM energy from the increasing wavelength of spacetime into positive gravitational matter. According to this principle, the negative EM energy of vacuum slowly lessens with distance inducing the lost into positive gravitational energy. The progressive deceleration of the space probe outward, interpreted as a constant sunward acceleration, is related to the deep general idea that the scalar field of the vacuum with an appropriate potential of negative energy is continuously able to transform its energy into a positive 
gravitational energy. It explains the anomaly of the outwards redshift in relation with the inward blueshift.

In a general sense, the EM wavelength of spacetime is assimilated with the cosmological constant interpreted as dark energy and corresponding in our theory to the negative mass-energy containing the baryons at speed under $c$ and the bosons at speed $c$. In a special sense, the EM wavelength of spacetime is associated with the energy and with the bosons at the speed of light.

The situation of the cosmological photons streaming from this special EM wave of spacetime, related by $\mathrm{CP}$ to the gravitational photon of the structure of condensation, is illustrated in a sort of double vacuum wells which forms a triangle 4 with $A$ at left, $E$ down and $G$ up. $A$ has a double role: it is the EM charge source for the EM cosmological photon on the structure of expansion (similar to the metric of special relativity) and it is the attractive mass for the gravitational photon on the structure of condensation (similar to the metric of GR). The branch $A G$ is of a gravitational well. The branch $A E$ belongs to an empty quantum well. Both wells are different but related.

When cosmological EM photons in space fall from $A$ to $E$ into the steady slowly growing vacuum which forms a "quantum well", they lose energy (redshift). The energy lost at point $E$ induces energy in gravitational photons on the structure of condensation at point $G$ falling down toward $A$ into a "matter well". So the gravitational photons rolling down the potential well will have an extra energy (blueshift) coming from the vacuum energy, adding the kinetic energy to a standard gravitational field obeying the EP. We can also say that the EM photon tumbling from $A$ to $E$ into a vacuum well becomes less energetic and slightly colder by $(v / c) T^{o}$, where the inferred velocity is $v<c$. The gravitational photon at $G$ recovers the energy lost by the opposite photon $E$, is hotter by $(v / c) T^{o}$, and is more energetic before he falls into this kind of gravitational well than the conventional gravitational photon gaining energy like a ball rolling down a hill.

When cosmological photons climb back out from $E$ to $A$, they are blueshifted, they gain EM energy. On the contrary, gravitational photons rolling up a matter well lose energy, are redshifted. It is like if the lost gravitational energy was transformed into EM energy. The deep meaning of CP is that when there is less EM mass-charge repulsive force in the structure of expansion going forward with the arrow of cosmological time, there is more mass-matter attractive force in the other structure. When there is more EM energy in the structure of expansion going backward against the arrow of cosmological time, there is more negative EM energy-mass, more quantum gravity, less classical gravitational energy-mass-matter, an antigravity. The conservation of mass-energy law is basically respected because there are two structures with two incompatible energies: the negative EM energy and the positive energy of rest mass. CP allowed their conversion in each other. The annihilation of one form of matter-energy is the creation of the other. The present universal current is the disintegration of the negative EM energy-mass converted into the positive gravitational energy-mass. 
With the theory of Relation, the cosmological photon is bound up with the gravitational photon. Thus, in the same proportion that the cosmological photon will be stretched and redshifted to a longer wavelength in the deepening potential well of the fabric of spacetime itself, the gravitational photon will be contracted and blueshifted to a shorter wavelength in a gravific potential well. This paradoxical situation gives the connexion between the compensation and the equivalence principles. Note that inflation or cyclic brane cosmology models, with the dark energy causing universal expansion to accelerate toward a big rip, are pure violation of EP.

\subsection{Macroscopic Casimir Effect: Induction of Gravity}

Photons of the interplanetary vacuum simply lose energy by traveling through space toward the infinity. In deep space there is an increase of wavelength of photon proportionally to the time/distance while passing through the space undisturbed [14]. The loss of energy induces a gravitational effect like a macroscopic Casimir effect [8] [9]. Gravity is induced when the EM energy of space is lowered between two plates. It implies a mechanical action which is equivalent to the ad hoc explanation saying that shorter external waves pushed plates one toward the other. It seems likely that redshifts may be due to a decelerating expanding EM spacetime transforming its loss of energy into the condensed matter of the gravitational structure. The observed excess redshift express both a steady decrease of the minimal energy of vacuum proportional to the distance and an induced gravity of matter. The observed excess blueshift express both a shorter distance and a higher frequency from positive quanta of energy.

Quantum vacuum field induced a constant gravity in fieldless space which is like the action of a constant gravitational field such as occurs at the immediate surface of a celestial body which, in accordance with GR, can be replaced by an acceleration of the observer with the same value $g$ directed opposite to the attraction. Pioneer effect is related to energy. Induced gravity by quantum vacuum aimed at the Sun means mass enlargement and lower gravitational potential (negative gravitational potential energy becomes smaller when bodies come closer) [15]. This anomalous lower potential energy from Sun is amount to an increase of mass (if $c$ is the speed of gravity and light, and if the distance is anomalously shorter, $M_{s}$ becomes more massive in $G M_{s} / c^{2}=r$ ). In fact, the $\mathrm{CP}$ is the underlying mechanism bringing a perpetual creation of positive energy-matter. Does it say there is no more conservation of energy and mass? Absolutely not, but this law must be enlarged to the notion of negative energy and mass in agreement with $\pm E= \pm\left(m c^{2}\right)$.

\subsection{Regarding a Lower Density of the Vacuum}

Anderson et al. [5] reported D.F.Crawford [16] suggesting a possible effect from applications of known physics: a gravitational frequency shift of the radio signals that is proportional to the distance to the spacecraft and the density of dust in 
the intermediate medium. In particular, he has argued that the gravitational interaction of the S-band radio signals with the interplanetary dust may be responsible for producing an anomalous acceleration similar to that seen by the Pioneer spacecraft. The effect of this interaction is a frequency shift that is proportional to the distance and the square root of the density of the medium in which it travels. Didon, Perchoux and Courten [17] proposed similarly that the effect comes from resistance of the spacecraft antennae as they transverse the interplanetary dust. This is related to more general ideas that an asteroid or comet belt, with its associated dust, might cause the effect by gravitational interactions or resistance to dust particles [18]. "However, these ideas have problems with known properties of the interplanetary medium: infrared observations rule out more than 0.3 Earth mass from Kuiper Belt dust in the trans-Neptunian region. Ulysses and Galileo measurements in the inner solar system find very few dust grains in the $10^{-18}-10^{-12} \mathrm{~kg} \cdot \mathrm{m}^{-3}$ range. The density varies greatly, up and down, within the belt (which precludes a constant force) and, in any event, the density is not large enough to produce a gravitational acceleration on the order of $a_{p}$ [5].

We think that the effect does not come from resistance of the spacecraft antennae as they transverse the interplanetary dust but on the contrary from a void of density. This is related to the more general idea that emptiness might cause the effect by gravitational interactions. It is not the resistance to "positive" dust particles but their absence which is generating gravity. Induced gravity in Casimir effect is not caused by presence but by absence of energy.

We suggest a possible effect from applications of unknown physics: a gravitational frequency shift of the radio signals that is proportional to the distance to the spacecraft and the loss of density energy (dust, etc.) in the intermediate medium. In particular, we argue that the gravitational interaction of the S-band radio signals with the growing empty space of the interplanetary vacuum may be responsible for producing drag acceleration similar to that seen by the Pioneer spacecraft. The effect of this interaction could be a frequency shift that is proportional to the distance and the square root of the "lack" of density of the medium in which it travels (not, as expected, proportional to the square root of the density of the interplanetary dust) [16].

It is assumed that the density of the vacuum $p_{\text {vacuum }}=p_{\text {solar system }} / n$ differs from the density in solar system (ecliptic) significantly. Index $n<1$ is the averaged characteristic of the negative energy layer thickness of which decreases by the law $l($ distance $)=v t$ (outwards) between the probe-transmitter and the Earth-receiver [19]. The Pioneer effect comes from resistance of the spacecraft antennae as they go through the interplanetary medium. This resistance, like associated gelatine, which is not real dust particles, is the negative pressure of a negative energy, which is the scalar medium of the vacuum. It induces a gravity which has a real mechanical effect similar to the Casimir effect. It is also presumed by the CP that this index ( $n$ toward 1 ) is likewise the averaged cha- 
racteristic of the positive gravitational energy layer thickness of which increases by the law $l=v t \quad$ (inward).

Having said this, we do not exclude the presence of dark matter as associated gelatine forming the resistance. Without elaborating on the nature of dark matter, it is in the theory of Relation an intermediate state between negative and positive energy-matter. The creation of this exotic matter is always accompanied with a creation of an exotic space, and both produces a gravitational flux density, both concerns non-baryons and non-bosons originating from the structure of expansion on a very slow way to become baryons and bosons in the structure of condensation. The specific case of Pioneer relates to bosons and "dark space" (void).

\subsection{Gravitational and Cosmological Photons}

The measurements allowed observing an increment of frequency of the radio signal sent off from the Earth and retransmitted by Pioneer 10. Light of frequency $V$ can be regarded, according to quantum theory, as consisting of quanta of energy $E=h v$. These have an inertial mass $m=E / c^{2}=h v / c^{2}$ and this is, according to the EP, equal to its gravitational mass. When light quanta $h c$ have travelled the distance $I$ toward the field of gravitation $g$, their energy has increased by $g / m$. The receiver on Earth detects a frequency

$$
h v^{\prime}=h v+g / h v / c^{2} .
$$

According to the $\mathrm{CP}$ in the deep space, we must add an extra induced gravity links up to vacuum. The gravitational interaction is caused by the focusing of the signal photons in curved space; in this case, the curvature is related by an anomalous induced gravity characterized by the lack of density of the interplanetary positive dust. When light quanta $h v$ have travelled the distance $I$ toward the field of gravitation $g$, their energy has increased by

$g / m+\Delta g / m$. Hence at the end of this journey the energy of quantum is:

$$
h v^{\prime \prime}=h v+\left(g / h v / c^{2}+\Delta g / h v / c^{2}\right) .
$$

The receiver on Earth detects a frequency slightly higher than the one detected according to the EP $\left(h v^{\prime}=h v+g / h v / c^{2}\right)$. It is a blueshift for the "gravitational" photons on the structure of condensation. Radio-signals intervals are shorter. The spacecraft in deep space undergoes more frequency which is not increment of speed because there is no recession: data proved Pioneer 10 is slowing down going outwards. On the standpoint of the spacecraft going outwards, the frequency observed on Pioneer $\left(v^{\prime \prime}\right)$ is anomalously more diminished than the expected frequency $\left(v^{\prime}\right)$.

$$
v^{\prime \prime}=v^{\prime}-\Delta v^{\prime}=v\left[1-(v+\Delta v)^{2} / c^{2}\right]=v\left[1-(g+\Delta g) l / c^{2}\right] .
$$

The observed redshift is greater than the calculated one. So the extra $g$ from $\mathrm{gl} / \mathrm{c}^{2}$ is an outwards deceleration interpreted, with a change of sign, like acceleration toward Earth-Sun, which is an application of CP. 
What about "cosmological" photons on the expansion structure? In deep space they follow the EM expansionist arrow of Time. Their sources are the negative matter-energy at the beginning of our cosmological time. They are in a position of a gravitational photon (emitted by star or planet) going against the field of gravitation $g$ and losing energy with outwards distance. With regard to the EP and Doppler effect, when light quanta $h v$ have travelled the distance $l$ with an expansionist EM field, their energy has decreased by $g / m$ giving the frequency $h v^{\prime}=h v-g / h v / c^{2}$. In empty space, the photon loses also an additional energy because a downward trend of minimal energy with distance. Hence at the end of this journey, according to the $\mathrm{CP}$, there is an anomalous redshift and the receiver on Earth should detect a frequency slightly lower than the one expected [12] [13]

$$
h v^{\prime \prime}=h v-\left(g / h v / c^{2}+\Delta g / h v / c^{2}\right) .
$$

Another way to say this is that in the deep space out of our solar system the probe follows an increasing EM spacetime wave. We postulate that with distance in vacuum the minimal negative EM energy of expansion is slowly diminished. It is a real effect in addition to the usual "Doppler effect". There is an anomalous loss of energy in the vacuum against the EM source giving an anomalous redshift. The energy of a quantum alongside with Pioneer going outwards is only

$$
h v^{\prime \prime}=h v^{\prime}-\Delta h v^{\prime}=h v\left[1-(v+\Delta v)^{2} / c^{2}\right]=h v\left[1-(g+\Delta g) l / c^{2}\right] .
$$

This equation without $h$ is like (15), where an anomalous acceleration toward the gravitational source gives the same redshift. It is like if the EM energy was converted into gravitational resistance or acceleration toward matter. Pioneer 10 in the deep space of expansion slows down going outwards; the cosmological photon loses energy, which fuels the gravitational potential. According to the $\mathrm{CP}$, the energy lost by the cosmological photon $\left(-g / h v / c^{2}\right)$ is retrieved by the gravitational photon $\left(+g / h v / c^{2}\right)$. The observed redshift on Earth is higher than predicted, but the real effect is a blueshift. In the vacuum, "lost energy" is equivalent to "gain of gravity", "anomalous redshift" from light quanta is equivalent to "anomalous blueshift" from anomalous acceleration (induced gravity).

\subsection{About Time and Metric}

Theory of Relation discerns two real spacetimes: the gravific spacetime, or Einsteinian spacetime, associated with positive energy and condensation; and the EM spacetime, or Lorentz-Maxwell spacetime, associated with negative energy and expansion. And two distinctive times going from past to future: cosmic time for the gravific spacetime (cosmic time of GR); and the cosmological time for the EM spacetime. Cosmologic time goes along with macroscopic thermodynamic time (entropy). Considering the two structures, electromagnetism becomes the counterpart of gravitation in the macrocosm [20] [21].

Usually we consider cosmic time, atomic time and ephemeris time. Cosmic 
time is the time of GR: dilatation of time when space contracts with greater curvature. Atomic time is also called "proper time". Ephemeris time is associated with Kepler-Newton time. It is the uniform time of Solar system; planets around the Sun. In a standard GR where empty space is without energy there is an agreement between those times. Non-conventional Pioneer effect contradicts relativity theory.

There is equality between atomic time of DNS antenna, cosmic time, ephemeris time but not with cosmological time on which they are depending. EM time (link with absolute time of our universe) is still ignored because not only the positive energy but also the negative energy of the vacuum is not considered. The origin of the anomalous signals is related to this cosmologic EM time. Cosmological time of a clock in the scalar field of vacuum (negative scalar) is longer, because the EM wave of spacetime is longer with the distance and loses some negative energy (tired light).

Atomic time of a clock on probe Pioneer in vacuum is also longer because matter gains the same negative energy converted into positive energy. Atomic time of DNS antenna takes the coincidental point-time of a time clock accelerating toward Sun (blueshift) corresponding to an anomalous decelerating Pioneer outwards (redshift). This shorter atomic time is not the same as the longer "proper time" of the atoms of Pioneer in deep space. In this regard, there is two "atomic times".

The radio signal sent off from the Earth and actively retransmitted by the spacecraft constitutes a "clock" which is not in a constant relation with an atomic clock on Earth. Perturbed shorter intervals affect the "temporal length" of the wavelength of the spectral narrow color lines even if it does not change the type of atom emitting the light. This is a violation of the relativistic $\mathrm{ds}^{2}$ based on Minkowski metric in space which embodies an imaginary time and directly no EM field. Our interpretation is that "temporal length" of the atom depends of distance and of a Lorentz-Maxwell metric with an EM wave of spacetime producing the cosmological time of our universe.

Note that, on Earth, only the atomic clock in relation with Pioneer going outwards with an anomalous deceleration is affected by the change of frequency. It reflects an anomalous and constant modification of the state of the vacuum surrounding Pioneer. The usual atomic clocks on Earth and on the planets are influenced just by the physical or mechanical local conditions in the near limited domain in which is this clock [22]. The irregular deceleration outwards interpreted as an anomalous acceleration inward is not seen on planets and there is no perceived change in the frequency of the usual atomic clocks, because orbital planets are in a finite and stationary movement.

This being said, ephemeris time apparently does not change. But beyond Neptune, Pioneer 10/11 radiometric data indicated a constant skewing between predicted and observed Doppler shifts [23]. It is a blueshift: radial distance become shorter because an excess of gravity makes the range of Newtonian radius 
shorter (greater curve with GR). The time of ephemeris, which is Newtonian time, is shorter and so is the range disturbance of the proportional radius.

\subsection{Relation (Not Coincidence), Ether and Two Structures}

There is a relation between, on one hand, the anomalous acceleration of the probe toward the Sun $\left(-a_{p}\right)$ and the acceleration of the clock $\left(a_{t}=a_{p} / c\right)$, and, on the other hand, an anomalous decrease in acceleration outwards and the increase of EM wavelength in deep space.

We have postulated the existence of two structures going from past to future but oppositely directed in space: condensation with the positive energy of gravity and expansion with the negative energy of electromagnetism. In this respect, EM force on cosmic scale is a counterpart comparable to gravitation.

GR is a local structure of condensation with space as a gravific ether and matter as an EM field. Gravitation is associated by GR to properties of spacetime. With Einstein, the field of electromagnetism in space is not determined by the gravific ether and its links in the ether are secondary, almost nonexistent [24]. SR associated Maxwell's electromagnetism to properties of spacetime and, in our view, this association constitutes the second structure, the one of the global expansion, initiated by Lorentz-Maxwell with an EM ether field as space and the ordinary matter. This second structure, inspired by Dirac, is a negative ocean where the negative energy-matter is perpetually transformed into the positive matter-energy of our world. Searching a physical meaning for the negative energy in his equations, Dirac uses above all the passage of positive energy toward the ocean of negative energy. Relation theory considers the ocean of negative matter-energy as the matrix of our world and its "evaporation" into the space-time-matter of our positive world follows the recessional motion with the arrow of time from past to future. Both structures exist and are necessary to our universe but properties of each structure are of different nature. The so-called greatest blunder of Einstein was not about the cosmological constant but the denial of a second structure in our world. It was more a limitation curbing unification than a mistake, since "ether" was set aside.

We assume that Pioneer follows the Lorentz-Maxwell structure in which the arrow of cosmological and thermodynamic times is going outwards toward expansion and entropy. It gives a deceleration of clock time, because longer cosmological time corresponds to a deceleration outwards and an increase of EM wavelength in deep space. Pioneer going outwards was under steady lower frequency drift of about $6 \times 10^{-9} \mathrm{~Hz} / \mathrm{s}$ which equates to a clock deceleration of $2.8 \times$ $10^{-18} \mathrm{~s} / \mathrm{s}^{2}$. The identity with Pioneer deceleration in deep space is $+a_{t}=+a_{p} / c$ (sign + means $a_{p}$ is outwards). This corresponds to the observed anomaly using the Doppler effect: the redshift is more than the calculated and verified by the frequent position measurements using the time of travel of the radio signal.

In fact, the location and the motion of the space vehicles point out less velocity [14]. Analysis of Pioneer's radio-metric tracking data has consistently indi- 
cated at heliocentric distances the presence of a small Doppler frequency drift which is a blueshift. Anderson et al interpreted it as a constant sunward acceleration of spacecraft [25].

The radio-signal known as the Pioneer anomaly follows the structure of Einstein-Newton with the positive energy of gravity. This structure of condensation and the cosmological time are going toward the future with the same arrow of time $(\rightarrow)$, but both structures have opposite spatial direction. Cosmic time follows the spatial direction of condensation $(\leftarrow)$. In this respect, let's say that our universe is a spacetime with a spatio-temporal dynamics. Spacetime of the expansion structure is temporally flat (not completely) with a positive or negative curvature, while the spacetime of the condensation structure is spatially curve.

Pioneer going sunward under steady higher frequency drift of about $-6 \times 10^{-9}$ $\mathrm{Hz} / \mathrm{s}$ which equates to a clock acceleration $-a_{t}=-a_{p} / c=-2.8 \times 10^{-18} \mathrm{~s} / \mathrm{s}^{2} \quad$ (sign means inward). The acceleration of Pioneer, the acceleration of clock and the frequency are higher. It's a blueshift.

There is an interpenetration of both structures giving opposite results and connected by the CP. Expansion is the dominant structure with cosmological EM time and negative energy begetting the condensation structure with gravific space and positive energy. Let's take $-a_{t}=+a_{p} / c$ (negative and positive signs mean inward and outwards). Pioneer going outwards slows down by following the way of spacetime EM in growth. The inverse of a growing universal time gives $-a_{t}$ which is a lower drift, or lower clock acceleration, sunward ${ }^{\dagger}$ (see 3.7).

The underlying mechanism is that the energy of the negative EM spacetime wave of expansion loses of its energy with the distance so generating the positive energy of the gravity. Longer cosmological time gives a diminishing frequency with distance because the negative energy density of the vacuum scalar field is slowly decreasing by generating an induced gravity, like a Casimir effect. The negative energy lost by space wave is converted into positive energy or, in other words, quantum vacuum is inducing gravity. Equivalently, it gives an anomalous acceleration of clock time, a blueshift going inward. Blueshift means a shorter wave of cosmic time on the condensation structure. The velocity of Pioneer outwards slows down; the distance and the time of travel of the radio signal are shorter.

This relation is not coincidence but an obliged natural link between both structures, an everlasting exchange between negative and positive energies. They are related by the CP: lost energy of expansion is recuperated into positive energy of condensation. There is more gravity-energy in condensation, because with distance there is less energy in the EM wave of expansion. This anomalous gravity-energy is equivalent to the anomalous shorter distance. More than a simple coincidence, $-a_{t}=+a_{p} / c$ express a perpetual relation between condensation and expansion, between both energies. 


\subsection{Predictions}

The observations made by WMAP, as well as the high redshift supernova data, are usually explained by an accelerating expansion of the present universe. However, with the current quality of the supernova 1a data, the allowed parameter space is wide enough to accommodate the decelerating models as well [26]. Moreover, the satellite XMM-Newton of Agency's European Space X-ray observatory (ESA) has returned data about the nature of the universe indicating that the universe must be a high-density environment, in clear contradiction to the "concordance model" relying on the theory of inflation. In a survey of distant clusters of galaxies, the results of XMM-Newton revealed that today's clusters of galaxies are superior to those present in the universe around seven thousand million years ago. Such a measure also goes toward a decelerated expansion [27].

At this stage we can wonder if we do not make wrong road from the initial conditions, if we really understand the relation between masses and gravitation, if the official accreditation in 1998 of a positive cosmological constant from the observations of type 1a supernova was not premature and misinterpreted. We foresee a rectification in the future. We think, for reasons else than those required by the classic big bang and by the decelerating Einstein-de Sitter model, which used to be the favoured model before these observations, that the global expansion decelerates since the beginning. The deceleration of Pioneer in the deep space where the wavelength of spacetime grows is in itself an experimental proof of a decelerated world.

In the future, there will be inward a predictable less acceleration for the probe, less clock acceleration, less blueshift, less shorter time interval between signal pulsations $^{\dagger}$ (see 3.6). In other words, with the distance and the time outwards the anomalous acceleration inward will be weaker, unless there is a sudden attractive body or an unexpected pocket of lower vacuum level. A longer cosmological time corresponds to a declining anomalous acceleration toward Sun. What we call an acceleration of time toward Sun is a deceleration of time toward infinity; the acceleration of Pioneer toward Sun is a deceleration outwards, being astride upon a slowed down expansion associated with the EM spacetime wave.

Notice that a number of people have pointed out

$$
a_{H}=c H \rightarrow a_{p}=a_{t} c \rightarrow \sim 8 \times 10^{-10} \mathrm{~m} / \mathrm{s}^{2}
$$

depending on the value of $H$ (inverse of the Universal Time) or $a_{p}$ [5]. The use of the time of travel of the radio signal gives an additional redshift going outwards: lower frequency, longer wavelength. It showed that there is here no Hubble effect (recession: faster speed of the galaxies with distance) corresponding to Doppler effect. They are prone to conclude that there is no recession at all and no expanding universe because the Hubble redshift is distance caused.

Our view is that the redshift is linked with the EM wave of spacetime of the second structure. A longer spacetime wavelength, or "tired light" in immediate deep space, points out a deceleration of expansion going toward the future. 
There is no contradiction with Hubble discovered nebular redshift versus distance explained through recession as long as it is toward the past on the structure of cosmological time: to see weakly the distant galaxies implicates see earlier in the past and a faster speed toward the point of origin. This means that the universe does not accelerate toward the future, and that its speed at the beginning was almost the speed of light.

\section{PART 11}

TIRED LIGHT MECHANISM: THE ZPF THEORY AND THE PRINCIPLE OF COMPENSATION

\section{Tired Light Integrated into Theory of Relation}

Till now, relativity and Doppler effects went along very well to explain the shifting of spectral lines. When astronomers look at the spectrum of light from a star or galaxy, they see the precise wavelength of the narrow, colored spectral line telling scientists what type of atom has emitted light. The Doppler effect is the apparent change in the wavelength of sound or light caused by motion of the source, the observer, or both. Waves emitted by a moving object as received by an observer will be blueshifted if approaching, redshifted if receding. The change in frequency depends on the speed at which the object moves to or away from the receiver [24]. With relativity, if we suppose two stars $S_{1}$ and $S_{2}$ link up by a rigid stick, a monochromatic light sent from $S_{1}$ to $S_{2}$ and reflected from $S_{2}$ toward $S_{1}$ would have the same frequency than the one measured by a clock in $S_{1}$ : the number of wavelengths along the stick will be the same.

A radio signal sent from Earth to Pioneer 10 and retransmitted to Earth would not have the same frequency as that initially measured by a clock on Earth: the observation of an abnormal blueshift indicates a wavelength that disappeared along the supposed stick between them. On the other hand, there is a cosmological redshift which excludes the Hubble redshift with its distancelsource proportionality, interpreted as the recession of galaxies. With distance, the light around the probe seems as tired as its speed.

Pioneer effect is the first support to explain it by the concept of "tired light". One of the difficulties before was that there was no consistent mechanism known that could give a tired light effect: it was not related to transmission in a transparent medium. Data from Pioneer 10 give evidence that there is plasma of space slowing down the photons.

Tired light is a class of hypothetical redshift mechanisms that were proposed as an alternative explanation for the redshift-distance relationship. It was first proposed in 1929 by Fritz Zwicky [28] who suggested that photons might slowly lose energy as they travel vast distances through a static universe by interaction with matter or other photons, or by some novel physical mechanism. Since a decline of energy corresponds to an increase in light's wavelength, this effect would produce a redshift in spectral lines that increase proportionally with the distance of the source. The term "tired light" was coined by Richard Tolman in the early 
1930s [29]. Today this model is considered highly speculative even if it was a serious alternative intended to replace the competing cosmologies of the big bang and the steady state, which both assumed that Hubble's law is associated with the metric of expanding space.

In the theory of Relation, tired light becomes a mixture of big bang and steady state cosmologies. The redshift is due to recessional motion and the photons emitted by a nebula lose energy on their journey to the observer. Pioneer 10 going outwards loses some frequency. The Pioneer effect is important because it demonstrates that light in space cannot be explained purely as a wave phenomenon. Relativity and the Maxwellian theory of EM wave cannot explain any real scattered energy and any shift in wavelength. Light must behave as a stream of particles whose energy is proportional to the frequency in order to explain what a Compton scattering for the space seems. This dispersion of energy, like a Compton effect in space, implies discontinuity in space instead of continuity. If photons can impair energy through some interaction with the medium (a plasma) located between the source and the observer, it requires a different metric than the one used by relativity [30].

\subsection{Gravitation and Electromagnetism: Two Sides of One Force}

The EM force in the vacuum, shown above, arises from a proposal of Sakharov [31] based on the work of Zeldovich [32] in which a connection is drawn between Hilbert-Einstein action and the quantum vacuum [33]. This leads to a view of gravity as "a metric elasticity of space". There were some early pioneering attempts, inspired by Sakharov's conjecture, to relate gravity to the vacuum from a quantum-field-theoretical viewpoint. The first step in developing Sakharov's conjecture in any detail was the work of Puthoff [34]. Gravity is treated as a residual force in the manner of van der Waals forces. Unlike the Coulomb force, which, classically viewed, acts directly between charged particles, this interaction is mediated by extremely tiny propagating secondary fields created by the zero-point fluctuations (ZPF)-driven oscillations [35], and so is enormously weaker than the Coulomb force. Puthoffs attempt to derive a Newtonian inverse square force of gravity proves to be unsuccessful.

Our approach, based on the theory of Relation, considers our whole universe as a white hole estimated as around $10^{53} \mathrm{~kg}$. The radius for the positive energy-mass ( $G M^{\circ} / c^{2}=G E / c^{4}=R_{U}$ ), as long as it is a Schwarzschild radius, is also a gravific wave $t_{o} c$. The same radius is the cosmological EM wave of the negative-energy mass of the vacuum $\left(t_{o} c_{E M}=h / m c=h c / m c^{2}\right)$. This means that during the expansion, triggered by the big bang, the negative mass-energy of a negative universe turned into a positive universe with a positive mass-energy. Our universe would be part of a cycle universe. Dark matter would be a negative mass-energy waiting to be transformed into positive mass-energy.

In classical physics, empty spacetime is called the vacuum. In quantum me- 
chanics, the vacuum is the state of minimum energy, the "ground state" full of fluctuating quantum fields.

These quantum fluctuations, ZPF a zero point energy associated with any quantum field supposedly do not involve real particles, only virtual particles-antiparticles pairs produced by the vacuum; the particle and the antiparticle try to separate for a short period (allowed by the principle of uncertainty), but they quickly annihilate with each other and the pair disappears again [36]. Since there is infinite number of harmonic oscillators per unit volume, the total zero-point energy density is, in fact, infinite. Through the process of renormalisation some sense can be made of infinite quantities. As it is usually implemented, this yields a zero energy density for the standard quantum vacuum.

The theory of Relation postulates that the zero-point energy yields a non-zero energy density for the quantum vacuum. It is extremely difficult to observe these vacuum fluctuations, since there is no state of lower energy to which the vacuum can be compared. However, the "Casimir effect" is one situation in which its effects can be seen indirectly.

In 1948, the Dutch physicist H.B.G. Casimir predicted that two clean, neutral, parallel, microscopically flat metals attract each other by a very weak force that varies inversely as the fourth power of the distance between them. It was experimentally verified in 1958. It can be understood in the following way: the zero-point energy filling the vacuum exerts pressure on everything; in most circumstances, this pressure is not noticeable, since it acts in all directions and the effect cancels. However, the quantum vacuum has different properties between the two plates. Some of the zero-point vibrations of the EM field are suppressed namely, those with wavelengths too long to fit between the plates. So, the zero-point energy density between the plates is less than that of the standard vacuum, i.e. it is negative. From this it follows that the pressure outside is greater and hence the plates feel an attractive force.

We think that it is precisely because the zero-point energy density between the plates is less than that of the standard vacuum that this negative energy induces (allowed by the CP) more positive energy, which is attractive. Since the beginning, the ground state of the going on expansion loses constantly some energy, and real fluctuations transform negative energy into positive energy. Pretend there is only virtual vibrations is a quantum way to corroborate the classical empty spacetime and deny physical significance to the states of negative energy.

The vacuum EM zero-point radiation is very dim, and theorists concluded that gravitation appears to be a long-range interaction akin to the van der Waals force. We prefer to declare it similar to the electromagnetism, which is less restrictive and includes both forces Coulomb and van der Waals. Roughly speaking, all the forces involved in interactions between atoms can be traced to the EM force acting on the electrically charged protons and electrons inside the atoms. This contains the intermolecular forces between the individual molecules. 
On one hand, at the ZPF of the vacuum, the negative EM force of the vacuum appears to be itself a long-range interaction such as the van der Waals force because it has been diluted since the beginning: the EM charge weakens during expansion and looks like a residuum force. And, if gravity is treated as a residuum force, it is because inertial and gravitational forces alike arise due to an interaction between vacuum EM zero-point radiation and negative subatomic particles giving birth to ordinary matter [35].

On the other hand, Coulomb force, which, classically viewed, acts directly between charged particles, is enormously stronger than the gravitational force. We calculate a difference of about $10^{36}$ orders of magnitude between the EM and the gravitational forces between two protons in the atom like if, in microcosm, electromagnetism was the only force existing. Suppose two protons on a surface of a collapsing star due to gravity, so that at the end a little bit more than a Fermi separated them. Then the difference of strength between gravity and electromagnetism would be of the order of about 1 rather than $10^{36}$. Gravitation, in this view, appears to be a short-range interaction akin to the Coulomb force. Gravity could then be construed as the second side, opposite and attractive, of the EM force. We already said that since the beginning of our universe the EM negative-energy transforms itself into positive gravitational energy. To be consistent with our theory, if we take the EM force of the vacuum and go back (from present to past) to the big bang and, on the other hand, if the whole universe collapses gravitationally (going from present to future), we will have the same magnitude of force. Like if electromagnetism in the vacuum and gravitation between stars were the repulsive and attractive sides of one force with EM origin. This said for the order of magnitude, we do not allege that gravity is exactly the opposite replica of electromagnetism.

\subsection{Cosmological Constant}

For a few years observational advances have led to the conclusion that the present universe is dominated by an energy density with negative pressure. Cosmologists call it dark energy and made it a version of Einstein's cosmological constant a constant, unchanging energy that pushes space to expand.

Microwave background experiments as well as supernova results strongly support the proposition coming from inflationary models that space is flat. To equal the critical density in a spatially flat universe it requires the total mass/energy density. Observations of galaxy clusters tell us that the matter contribution (baryonic and non-baryonic) to the total energy density can amount to only $20 \%-30 \%$ of the needed critical density. Dark energy, which makes up about three-quarters of the energy density of the universe, accelerates cosmic expansion. With inflationary cosmology, the cosmological constant is driving this accelerated expansion only for a tiny fraction of a second. With quintessence model there is a slower and steady accelerated phase of spatial expansion. Whatever the model, a non-vanishing cosmological constant is expected, on the basis of quantum 
theory, to describe the astronomical observations [37].

Cosmologists are then confronted with two problems. First, since all sorts of vacuum energies contribute to the effective cosmological constant, why is the total vacuum energy density so small by all particle physics standards? Second, since the vacuum energy density is supposed to be constant in time while the matter energy density decreases as the universe expands, what can explains this cosmic coincidence that both densities are comparable just at the present time, while their ratio has been tiny in the early universe and will become very large in the distant future? [38]

Theory of Relation is not an inflationary cosmology and claims that the energy density of vacuum is negative with an associated positive pressure driving a decelerated expansion of empty space. It assimilates dark energy with the cosmological constant, and also with the negative energy, while it is generally perceived as "exotic" positive energy. Even if the assumption was right that a spatially flat universe needs about $30 \%$ of ordinary and dark matter and about $70 \%$ of dark energy to obtain the total mass/energy density equaling the critical density, we disagree with the interpretation that the expansion of the universe is accelerated. We think the observations, supernova and CDM, are construed through a very speculative inflationary cosmology. We conclude that the percent of positive matter will grow and that the percent of expansionist dark energy will wane until equality, it will be then the path toward a big crunch.

Cosmologists are not satisfied with identifying the cosmological constant with dark energy because the expected sea level for the quantum vacuum is much higher than we observe: naively one should indeed have a characterless universe, with matter drowned by $\sim 122$ orders of magnitude below the energy density of the cosmological constant. But the cosmic concordance measures only a factor of a few differences. Furthermore, the matter and radiation we see in the universe evolves with the expansion, while the cosmological constant does not [37]. What is the explanation? The theory of Relation assumes that there is more a causal relation than a scheming coincidence encloses in the question "why both densities without apparent relation are today about the same order"? His approach is to change the initial conditions: the energy density of the cosmological constant was large at the beginning and composed of baryons and bosons which gave up their energy to become actual "tired matter" and "tired light". On this temporal mode, the primordial vacuum energy was $\sim 122$ orders of magnitude greater than the present nearly zero of the cosmological constant. The matter and light of our universe came from the transformation of the negative energy-matter into positive energy-matter throughout expansion. The energy of the vacuum is dynamic; its density varies in time: that is why the density of the global positive matter diluted into the expansionist void coincides with the density of the vacuum [39] [40].

All this negative energy is now recycled into positive matter in the framework of GR with four dimensions, with matter-galaxies attached to the fixed grid on 
the expanding fabric of spacetime, like dots on the surface of a balloon. Density of matter of the whole universe is very weak, nearly zero, but would be as big as initial negative energy if the mass distributed throughout the surface was concentrated in a pre-relativistic Newtonian centre. On this spatial mode, the small, dense vacuum would produce a higher energy of 122 orders of magnitude. Subsequently, the spatial radius of the universe would have the same variation as the temporal wave of spacetime expansion: $R_{u}=t_{o} c_{e m}$.

About $4 \%$ of matter is ordinary (baryonic: $\sim 0.4 \%$ visible, $\sim 3.6 \%$ not visible), and we speculate that dark matter is a negative matter which is still waiting to be transformed into positive ordinary matter. The very low dark energy, taking the form of a cosmological constant, is like a cold radiative surface on a featureless negative Dirac's ocean. A low vacuum energy keeps evaporating, which dulls the negative EM energy of our second structure, and in the same breath enlarges the positive energy, which allows the gravitational charge to take the ascendant. It is the deep explanation of the tired light theory.

Note that "quintessence" is dynamic, and has a density of energy that varies through time, like the negative EM spacetime wave. Quintessence is a hypothetical form of dark energy postulated as an explanation for observations that infer an accelerated universe; it is a positive energy density of the vacuum which proceeds from a positive cosmological constant with a negative pressure. By contrast, the negative EM spacetime wave of the theory of Relation has a negative energy density of vacuum resulting from a negative cosmological constant in decline, which implies a positive pressure that requires a decelerated expansion of the universe.

\subsection{Principle of Compensation and the Zero-Point Fluctuations}

In order to solve the "unsolved mystery in modern physics", known as inertia (the instantaneous opposition to acceleration of all material objects), Haisch and Rueda related EP to Sakharov's conjecture of a connection between Einstein action and the vacuum [41] [42]. They said that the properties of the EM quantum vacuum as experienced in a Rindler constant acceleration frame were investigated, and the existence of an energy-momentum flux was discovered and also its relative radiation, both stem from event-horizon effects in accelerating reference frames. The force of radiation pressure produced by the energy-momentum flux proves to be proportional to the acceleration of the reference frames, which leads to the quantum vacuum inertia hypothesis meaning that the inertia of matter could be interpreted at least in part as a reaction force originating in interactions between the EM zero-point field and the elementary charged constituents (quarks and electrons) of matter. They tried to demonstrate that this approach to inertia is consistent with GR and it appeared also that Newton's equation of motion ( $f=m a$ ) could be inferred from Maxwell's equations as applied to the ZPF, i.e. the stochastic electrodynamics (SED) version of the quantum vacuum [43]. 
Their goal was to show that a ZPF-EM reaction force will prove to be the exact opposite of this, and can therefore reasonably be interpreted as the inertia of the object, i.e. that in general [41] [44]

$$
f^{z p}=f_{r}=-f .
$$

While we view their approach as promising we propose that the link of inertia of material objects with the hidden structure of the vacuum pass by both $\mathrm{EP}$ and CP. To illustrate this let us take a simple and gross comparison from the theory of Relation with two structures.

Imagine that the negative and expansionist EM wave of spacetime in the second hidden structure of the vacuum is represented by a rocket $\mathrm{A}$ going toward infinity. The two fuels of the rocket are mixed in the combustion chamber, where they undergo a violent chemical reaction. The products of this reaction are hot, high pressure gases that escape through the exhaust nozzle with a high velocity and thereby acquire a large backward momentum. The total momentum of the system must remain constant and so the backward momentum acquired by the ejected gases must be compensated by an equal and opposite forward momentum given to the rocket and unspent fuel: $p_{r(A)}=-p_{(A)}$. In terms of force: $f_{r(A)}=-f_{(A)}$. The reaction force $f_{r(A)}$ is EM and it is the asymmetric energy and momentum flux at a non-zero-point-field ZPF, so this back-reaction of lost EM energy is also a zero-point force ZPF called the inertial reaction:

$$
\begin{gathered}
f_{r(A)}=f_{(A)}^{z p f} . \\
-\left(-f_{(A)}=f_{r(A)}=f_{(A)}^{z p p}\right) .
\end{gathered}
$$

Imagine also that the first structure of positive gravific spacetime is represented by a similar rocket B in gravity-free space going in opposite direction; that some sort of connecting pipe (or wormhole), can collect the backward negative energy and momentum flux of $A$, and transform it into a positive energy which is conveyed to $\mathrm{B}$. With this momentum, $\mathrm{B}$ will accelerate $(f=m a)$ toward star and will have an equal and opposite reaction in direction of $A:-f_{B}=f_{r(B)}$ $\left[(f=m a) ; f=-f_{r}=-(-m a) ; f_{r}=-m a=-f\right]$. In this case we can say that the back momentum of $\mathrm{B}$ in direction of $\mathrm{A}$ is equal to the back momentum of $\mathrm{A}$ in direction of $\mathrm{B}: f_{r(B)}=f_{r(A)}$, giving

$$
+\left(-f_{(B)}=f_{r(B)}=f_{(B)}^{z p f}\right) .
$$

This equivalence of reactions gives apparently a connection between Einstein's EP and the inertia of the vacuum based on Sakharov's conjecture.

In the case of stationary orbit, there seems no difference between positive or negative energy, like if there was just one structure with no vacuum energy

$$
\left[-\left(-f_{(A)}=\overrightarrow{f_{r(A)}}=f_{(A)}^{z p f}\right)=+\left(-f_{(B)}=\widetilde{f_{r(B)}}=f_{(B)}^{z p f}\right)\right] .
$$

There is a balance between $F_{g}$ and $F_{i}$, and the ZPF which is usually perceived as isotropic, symmetric, stationary or uniform-motion frame, without 
resistance. The EP is safe but does not explain the lost energy of A gained by $\mathrm{B}$.

In deep space, $\mathrm{A}$ and $\mathrm{B}$ are in a dynamical system. With time and distance, a kind of resistance not coming from particles of positive energy settles in front of A, which causes an unexpected loss of energy and a decrease in speed. It follows that $B$ receives additional energy and has an abnormal acceleration. After a while, the deceleration of A became smaller, as did the acceleration of B. The EP explains neither this anomaly nor the change of sign of the energy.

We consider that Pioneer in deep space is a material object in a dynamical system which undergoes a change of its velocity with respect to time on both interpenetrated structures. The tired light wave of spacetime causes an asymmetric non-zero-point field of the quantum vacuum which decelerates the advancing probe. Tired light phenomenon is analogously the rocket A which loses energy going toward infinity.

According to the strong equivalence principle (SEP), in a zero homogeneous background of ordinary classical electrodynamics, inertial mass is equal to active and passive gravitational mass $\left(m_{i}=m_{g-a}=m_{g-p}\right)$. Ordinary classical electrodynamics presupposes positive energy solutions and, accordingly, negative energy is merely Dirac's artifice, a virtual particles background that not changes the laws of physics when exchanging one inertial reference system for another. In a non-zero background SEP could be satisfied if

$$
\overrightarrow{m_{g} a}=\overrightarrow{m_{i} a}=\overrightarrow{m_{z p f}} a \text {. }
$$

It would be operational if at least there was equality between the coefficients of resistance $m_{g}$ and $m_{z p f}$. This would require more dust with positive energy in deeper space which is not the case $\left(m_{z p f} \neq+E_{z p f} / c^{2}\right)$.

On the contrary, the space vacuum density is less positive. If the ZPF force represents the system of inertia (resistance to positive energy), then there is less inertia than gravity, which is construed by the theory of Relation as a reduction in negative EM energy converted into positive gravitational energy. At point $F_{Z P F}$, the declining negative energy flux $\left(-F_{Z P F}=-E_{Z P F} / c^{2}\right)$, characterized by more empty space, interacts with quarks and electrons in the physical probe, adds a growing positive energy $\left(+E_{\text {zpf }} / c^{2}\right)$, and generates an inertia reaction force in the opposite direction. The theory includes the real, not just virtual, effects of the quantum vacuum EM in physics, and CP allows the conversion of the energies that induce weight. The anomalous deceleration of Pioneer outwards is interpreted like an anomalous acceleration toward the Sun, giving

$$
f_{(P)}=-f_{r(P)}=-f_{(A)}^{z p f} \text {. }
$$

There is an anomalous acceleration toward the Sun in relation with an equal deceleration outwards, an imbalance between $\overleftarrow{m_{g-a}}$ and $\overrightarrow{m_{i}}$, with $m_{g-a}>m_{i}$. By considering Pioneer as an integral part of a freely falling local frame in a flat space-time, an "abnormal" influence is measurable from the gravitational potential which causes an acceleration of the frame as a whole. The outcome of a local non-gravitational test experiment is dependent of the velocity and location of 
the freely falling frame relative to other matter in the universe [45] [46] [47].

Relatively to Newton's third law, which requires that the driving force defined in the second law be counterbalanced by a reaction force traditionally called the inertia of matter [44], the meaning is that the abnormal acceleration of Pioneer toward the Sun is counterbalanced by the deceleration of the expansion in opposite direction.

The Pioneer's anomaly brings an argument against the equality (or proportionality) of inertial mass to gravitational mass within the standard theoretical framework of GR. The energy flux at point $F_{Z P F}$ causes asymmetry in the metric: the gravitational system toward Sun gains energy $\left(+f_{(B)}^{z p f}\right)$ while the inertial system outwards loses the same energy $\left(-f_{(A)}^{Z p f}\right)$. Equation (22) becomes an inequality

$$
\left[-\left(-f_{(A)}=f_{r(A)}=f_{(A)}^{z p p}\right)-\Delta f_{(A)}^{z p f}\right] \neq\left[+\left(-f_{(B)}=f_{r(B)}=f_{(B)}^{z p f}\right)+\Delta f_{(B)}^{z p p}\right] .
$$

It also exhibits two opposite systems, like two structures going in the opposite infinity but in close relation, one springing from the other.

In virtue of the $\mathrm{CP}$ there is an induced gravitation at the point ZPF: negative EM energy of the expansion converts its energy into positive gravitational condensable energy. This is reflected in a compensation which requires that the EM background has a spectrum of variable Lorentz energy density and real vacuum interactions. In that sense, both energies are equivalent to give birth to inertia.

Both principles are complementary and are necessary to understand our dual universe: $\mathrm{EP}$ for the global positive matter; $\mathrm{CP}$ for the universal negative expansion. An observer on the first structure (positive) will have the trend to adopt Mach proposition saying that inertia, the instantaneous opposition to acceleration of all objects, somehow originated in a global linkage of all matter in the universe. An observer on the second structure will adopt the view of Newton that inertia is an inherent property of matter for which a further explanation possible is a link with an absolute space. For Mach, matter is inertia. For Newton, space is inertia. Mach's explanation is for the positive energy while the one of Newton is for the negative energy. The theory of Relation embraces both propositions: absolute space of Newton is the negative EM energy of the expansion converted into the positive energy of matter.

\subsection{Pioneer Effect and Theory of Quantum Gravity}

If anomalies in various stellar and galactic redshifts, commonly interpreted as Doppler shifts in an expanding universe, are in fact the result of loss of energy by observed photons traversing a radiation field (Erwin Finlay-Freundlich)), where does this loss of energy go? No generally accepted physical mechanism has been proposed for this loss, which seems against the conservation of energy, and some conclude that the assumption of a universal expansion is erroneous [48].

The theory of Relation says that photons slowly lose energy across an expanding world, thus creating an EM space-time. Since a fall of energy corres- 
ponds to an increase in cosmological wavelength of light, this effect would produce a redshift in the spectral lines which would augment proportionately with the distance of the source. The energy lost by the photons of the EM cosmological wave of spacetime reappears in gravific spacetime. If we state that there is an EM negative structure of the expansion directly connected with the positive gravitational structure, by which unknown physical mechanism in the vacuum the lost negative energy could be converted into positive energy?

The theory of Relation is in the line of those who support the ZPF proposal seeking to ascribe gravitation entirely to interaction with zero-point radiation. Puthoff s gravity [34] [49], suggesting that gravitation is a residual effect of the ZPF of the vacuum EM field, is positive-energy "pushing together" gravitation, similar to Lesage's "ultra-mundane corpuscles". We prefer a model proposed by Hotson [8], which also utilizes a residual effect of electromagnetism, whereas negative-energy forces act by "pulling together" [35]. This attraction suggests that the zero-point energy is felt by the Casimir effect. Two metal plates brought sufficiently close together, allowing only small, high-frequency EM "mode" of the vacuum energy to squeeze in between, will attract each other very slightly [50]. Frequencies of any type of EM mode (which give more fluctuations) outside of the plates creates a vacuum pressure which brings the two plates closer to each other. Our explanation is to say that empty space with longer wavelengths induces gravity. There is attraction when the energy is thrown out of the space which separates the plates, the energy then becomes lower than that of the standard vacuum. We assimilate this phenomenon to a kind of gravity illustrating the CP: the less energy there is in empty space, the more gravity there is.

If gravity pulls the plates together, how comes? By what mechanism if the negative energy acts? It would take a quantum theory of gravity involving the idea of the "graviton" as the quantum of the gravitational field to answer these questions. Unfortunately, it is not easy to construct theories of physics in vacuo. Physicists feel that attempt to formulate a theory of quantum gravity to incorporate GR and quantum mechanics in a fundamental way must require the input of radically new concepts and quantic language of gravitation. In spite of a variety of a priori assumptions, arguments and disagreements, not only there is no experience to test a theory of quantum gravity but there is not even a reasonable existing theory [51]. The absence of any experimental input opens up a Pandora's Box of possibilities. One approach, which remains elusive, is to regard gravity as "just another field", and to treat it in the way that has been successful with the EM interactions. Our view is that Pioneer effect could be a kind of experimental input that might provide a consistent theory of quantum gravity.

In 1937, Louis de Broglie wrote: "The neutrino would be a kind of half-photon. Isolated, that is to say not accompanied by an antineutrino, it would have no electromagnetic field since it could not annihilate itself by photoelectric effect. But united with an antineutrino, it would form a photon 
and would possess an electromagnetic field of Maxwellian type" [52] [53]. Gamow [54] and Niels Bohr emitted the possibility of a link between neutrino and graviton. In the early 1950s, Erwin Finlay-Freundlich proposed a redshift as "the result of loss of energy by observed photons traversing a radiation field" [55]. R.A. Alpher noted "No generally accepted physical mechanism has been proposed for this loss", though P.F. Brown proposed that the energy lost reappears as neutrino pairs resulting from the exchange of a graviton between two photons.

For our part, we think that the empty space created by the evacuated energy between the Casimir plates is filled with gravitons which can be created by neutrinos. We suggest that "the neutrino is a kind of half-graviton. Isolated, that is to say without an antineutrino, it would have no gravitational field since it could interact just with weak nuclear force. But linked to an antineutrino, it would form a graviton and would possess a gravitational field of Casimir type" (The square root of a negative number provides a negative solution and, in this case, a real negative energy solution)

$$
\begin{aligned}
& \text { neutrino \& antineutrino } \rightarrow \text { graviton } \\
& 10^{-34} \mathrm{~kg} \times 10^{-34} \mathrm{~kg} \rightarrow 10^{-68} \mathrm{~kg} \\
& \left(10^{-68}\right)^{1 / 2} \mathrm{~kg} \times\left(10^{-68}\right)^{1 / 2} \mathrm{~kg} \rightarrow 10^{-68} \mathrm{~kg} \\
& 1 / 2 \text { graviton } 1 / 2 \text { graviton }
\end{aligned}
$$

This assumption of a graviton composed of two half neutrinos is based on a connexion between Casimir experience, the Hubble constant, the Pioneer effect, and also with the age and the radius of the universe.

Firstly, about the Casimir experience, S. K. Lamoreaux, while at the University of Washington, conducted the most precise measurement of the Casimir effect [50]. Helped by his student Dev Sen, he used gold-coated quartz surfaces as his plates. One plate was attached to the end of a sensitive torsion pendulum; if that plate moved toward the other, the pendulum would twist. A laser could measure the twisting of the pendulum down to 0.01 micron accuracy. A current applied to a stack of piezoelectric components moved one Casimir plate; an electronic feedback system countered that movement, keeping the pendulum still. Zero-point energy effects showed up as changes in the amount of current needed to maintain the pendulum's position. Lamoreaux found that the plates generated about 100 microdynes (one nanonewton: $10^{-9} \mathrm{~N}$ ) of force. The result falls within $5 \%$ of Casimir's prediction for that particular plate separation and geometry. The strength generated by the plates is

$$
h c / \lambda^{2}=10^{-9} \mathrm{~N},
$$

so $\lambda=1.41 \times 10^{-8} \mathrm{~m}$. Energy is

$F \lambda=\left(10^{-9} \mathrm{~N}\right)\left(1.41 \times 10^{-8} \mathrm{~m}\right)=\sim 1.4 \times 10^{-17} \mathrm{~J}=\sim 88 \mathrm{eV}$. The force of the Casimir experience moving a plate toward the other comes into existence from the energy of quantum fluctuations, from physical properties of the vacuum [56].

Secondly, if we suppose that the configuration of all the mass in the universe, 
estimated $\sim 2 \times 10^{53} \mathrm{~kg}$, is concentrated at the center of a sphere with radius $\sim 10^{26}$ $\mathrm{m}$, we note about the same Casimir strength. The force between the global mass and a peripheral body of $1 \mathrm{~kg}$ would be $\sim 10^{-9} \mathrm{~N}$ :

$$
F=G M m / R^{2}=\left(2 \times 10^{53} G \times 1\right) /\left(10^{26}\right)^{2} .
$$

The gravitational acceleration toward the center would be $\sim 10^{-9} \mathrm{~m} / \mathrm{s}^{2}$.

Thirdly, the cosmological acceleration $\sim 8 \times 10^{-10} \mathrm{~m} \cdot \mathrm{s}^{2}$, noted before (18), is also the reported Sunwards anomalous acceleration of Pioneer 10 [5]. There is a correlation between 1) the acceleration of expansion with Hubble constant based on the age of the universe $(H=1 / t), 2)$ the anomalous acceleration of the spacecraft, and 3) the acceleration of time [57]. On one hand, 2) and 3) have the same acceleration and force in common, and are roughly on the same "cosmological" scale. On the other hand, even if the three items have the same force $\left(10^{-9} \mathrm{~N}\right)$, the microscopic scale of Casimir force and the macroscopic scale of the universe have apparently no rational classical link. With the theory of Relation there is a deep and strong link. Both effects, Pioneer and Casimir, happen in empty space, at the zero-point energy. Concerning Pioneer effect, we have already put a link between the negative EM cosmological wave of spacetime losing energy and the gravity which induces matter. In the case of Casimir, the effect happens after a void is created between the plates. We assume that the expulsion of the positive energy which creates a void gives way to the negative scalar EM cosmological wave of spacetime which is on the same scale as 2) and 3) (i.e. the EM cosmological wave equivalent to the radius of our universe.). Since there is almost the same strength between the anomalous acceleration of the Pioneer effect and the Casimir effect in empty space, we presume that the void in Casimir experience is about the same magnitude as that of the vacuum enveloping the spacecraft. At this zero-point energy, there is an ocean of photon and electrons and, also, of gravitons and neutrinos.

The force $\sim 10^{-9} \mathrm{~N}$ corresponds to a particle $\sim 10^{-34} \mathrm{~kg}(\sim 88 \mathrm{eV})$ considered as a possible mass for the neutrinos. Our proposition for the neutrino to be half a graviton gives for the graviton

$$
\left(\sim 10^{-34} \mathrm{~kg}\right)^{2}=\sim 10^{-68} \mathrm{~kg} ;\left(\sim 10^{-33} \mathrm{eV}\right) .
$$

This weight matches with the one assessed for the graviton particle [58], if we assimilate the negative EM cosmological wave with the radius of the universe (based on Schwarzschild: $R_{S C H}=t_{o} c=G M / c^{2}$ ); $t_{o} c$ is then like a Compton wavelength of the quantum graviton for the radius of the universe

$$
\lambda_{g}=h / m_{g} c=c / H=t_{o} c=R_{U}=\sim 10^{26} \mathrm{~m} .
$$

The value $\sim 10^{-68} \mathrm{~kg}$ for $m_{g}$ could also be for a Compton wavelength of the quantum photon for the radius of the universe

$$
\lambda_{\gamma}=h / m_{\gamma} c=c / H=t_{o} c_{E M}=R_{U}=\sim 10^{26} \mathrm{~m} .
$$


Note that $m=\sim 10^{-68} \mathrm{~kg}\left(\sim 10^{-33} \mathrm{eV}\right)$ could be a quantum graviton, or photon, and the dimensionless coupling constant for the universe could be $G M_{\text {graviton(or photon) }}^{2} / \hbar c=\sim 10^{-122}$. The inverse is the cosmological constant: $=\sim 10^{122}$ [59].

Pioneer effect, tired light, and the postulated graviton resulting from neutrinos pair in the framework of the theory of Relation, could provide a basic idea of what we simply do not know.

\section{Conclusions}

General Relativity deals with the interaction between a system in inertial space and gravity-matter based on the principle of Equivalence. The theory of Relation considers it like its first structure of "condensation" with a positive matter-energy accompanied by an empty space generally ascribed without gravitation or energy. This structure is directly counterbalanced by a second one with a negative matter-energy driving the expansion. Since the so-called big bang, a negative electromagnetic mass-energy transforms itself into the positive gravitational mass-energy. We live in a dualverse rather than a universe, where the parameters of both structures are not independent but linked by a communicating vessels system, such as all that is earned by one is lost by the other one. One of the consequences is a deceleration of the expansion in phase with a local increment of gravity of the galaxies. Since 1998, a logic and over-simple line of reasoning based on a positive dark energy of empty space, interprets wrongly, in our opinion, the global expansion as accelerated with a positive cosmological constant.

The Pioneer anomaly in deep space is the first to reveal a decreasing negative electromagnetic wave of tired light which induces a positive gravity in the vacuum, by virtue of the postulated principle of Compensation. We think that other anomalies, like "Allais effect" [60] [61] or "galaxy rotation curves" [62], can also be explained by the theory of Relation, which is in the frame of "cyclic universe theory".

In the deep space outside our solar system, the probe is in a flat space (not in a Hubble space with recession), in the furrow of an expansible electromagnetic wavelength of spacetime. With the distance in vacuum, the minimal negative electromagnetic energy slowly lowers which induces the positive gravitational energy. In this induction process, cosmological photons gradually lose energy, thus heightening the gravitational potential. It is like if the electromagnetic energy was converted into gravitational resistance or acceleration toward matter. Pioneer 10 slows down going outwards and the observed frequency of the redshift on Earth is lower than predicted. Pioneer effect is an experimental "violation" of the principle of Equivalence. Such a violation shakes the foundation of physics. That does not mean that Einstein's theory is fundamentally "wrong" but incomplete, that the gravitational interaction is more complex than previously assumed, and contains, in addition to the interacting Einsteinian field of spin 2, 
the effect of another long-range field, which is that of the second structure which incorporates an electromagnetic spacetime. This new vision of the universe is based on the Compensation principle allowing interaction between material system in deep space and vacuum electromagnetic-zero-point fluctuations inducing gravity. The equivalence principle and the compensation principle coexist and are complementary.

\section{Conflicts of Interest}

The author declares no conflicts of interest regarding the publication of this paper.

\section{References}

[1] Anderson, J.D., Laing, P.A., Lau, E.L., Liu, A.S., Nieto, M.M. and Turyshev, S.G. (1998) Physical Review Letters, 81, 2858-2861. https://doi.org/10.1103/PhysRevLett.81.2858

[2] Turyshev, S.G., Anderson, J.D., Laing, P.A., Lau, E.L., Liu, A.S. and Nieto, M.M. (1999) The Apparent Anomalous, Weak, Long-Range Acceleration of Pioneer 10 and 11. 34th Rencontres de Moriond Meeting on Gravitational Waves and Experimental Gravity, Les Arcs, 23-30 January 1999, 1-7. https://doi.org/10.2172/353450

[3] Anderson, J.D., Lau, E.L., Turyshev, S.G., Laing, P.A. and Nieto, M.M. (2002) Modern Physics Letters A, 17, 875-885.

[4] Mbelek, J.P. (2003) The Pioneer Anomaly: A Bulk Scalar Field? Service d'Astrophysique, C.E. Saclay F-91191 Gif-sur-Yvette Cedex, 13.

[5] Anderson, J.D., Laing, P.A., Lau, E.L., Liu, A.S., Nieto, M.M. and Turyshev, S.G. (2002) Physical Review D, 65, Article ID: 082004.

[6] Markwardt, C.B. (2002) Independent Confirmation of the Pioneer 10 Anomalous Acceleration.

[7] Dittus, H., Turyshev, S.G., Lämmerzahl, C., Theil, S., Foerstner, R., Johann, U., Ertmer, W., et al. (2005) A Mission to Explore the Pioneer Anomaly. Proceedings of 39th ESLAB Symposium, Noordwijk, 19-21 April 2005, 1-9.

[8] Hotson, D.L. (2002) Dirac's Equation and the Sea of Negative Energy. Infinite Energy, Issue 44, Part 1, 10-12, Part 11, 2, 6, 19-22.

[9] Hatch, R.R. (1999) Electrodynamics, 10, 69-75.

[10] Einstein, A. (1961) Relativity. Three Rivers Press, New York.

[11] Ostermann, P. (2003) Relativity Theory and a Real Pioneer Effect.

[12] Born, M. (1962) Einstein's Theory of Relativity. Dover Publications, Inc., Mineola, 121-125, 216, 339, 352-353.

[13] Rocard, J.-M. (1986) Newton et la Relativité, Que sais-je? Presse universitaire de France, 103.

[14] Stolmar, A. (2001) Pioneer 10 Anomalous Acceleration Is Distance Caused Hubble Red-Shift. http://sci.tech-archive.net/Archive/sci.physics/2006-01/msg00766.html

[15] Atkins, K.R. (1970) Physics. J. Wiley \& Sons, Inc., Hoboken, 130.

[16] Crawford, D.F. (1999) A Possible Explanation for the Anomalous Acceleration of Pioneer 10. 
[17] Didon, N., Perchoux, J. and Courtens, E. (1999) Université de Montpellier.

[18] Nieto, M.M., Turyshev, S.G. and Anderson, J.D. (2005) Physics Letters B, 613, 11. https://doi.org/10.1016/j.physletb.2005.03.035

[19] Tcherepanov, O.A. (2002) Doppler-Mikhelson's Principle and a Pseudacceleration of the Nasa's Spacecrafts: Pioneer 10 and Pioneer 11 Have Discovered in the Perihelion Space the Faintly Refractive Medium.

[20] Ratcliffe, H. (2005) Progress in Physics, 3, 19-24.

[21] Thuan, T.X. (1995) Un Astrophysicien. Champs Flammarion, Paris, 76-83.

[22] Dive, P. (1945) Les Interprétations Physiques de la Théorie d'Einstein. Dunod, Paris, 52.

[23] Renshaw, C.E. and Kallfelz, W.L. (1998) Google Scholar, Physics Essays, 11, 361-366.

[24] Einstein, A. (1972) Réflexions sur l'électrodynamique, l'éther, la géométrie et la relativité. Gauthier-Villars Éditeur, 73, 113-114.

[25] Johann, R., Dittus, H. and Lämmerzahl, C. (2007) Exploring the Pioneer Anomaly: Concept Considerations for a Deep-Space Gravity Probe Based on Laser-Controlled Free-Flying Reference Masses.

[26] Zwicky, F. (1929) PNAS, 15, 773-779. https://doi.org/10.1073/pnas.15.10.773

[27] Hubble, E. and Tolman, R.C. (1935) Astrophysical Journal, 82, 302. https://doi.org/10.1086/143682

[28] Pecker, J.-C. and Vigier, J.-P. (1986) A Possible Tired Light Mechanism. Observational Cosmology, Proceedings of the IAU Symposium, Beijing, 25-30 August 1986, $507 \mathrm{p}$. https://doi.org/10.1007/978-94-009-3853-3 48

[29] Tolman, R.C. (1934) Relativity, Thermodynamics and Cosmology. Dover Books, Mineola, 287.

[30] Misner, C., Thorne, K. and Wheeler, J.A. (1971) Gravitation. Freeman, New York, 426-428.

[31] Sakharov, A.D. (1967) Doklady Akademii Nauk SSSR, 177, 70-71. (English Translation (1968) in Soviet Physics-Doklady, 12, 1040-1041).

[32] Zeldovich, Yu.B. (1967) Zh. Eksp. \& Teor. Fiz. Pis'ma, 6, 883-884. (English Translation (1967) in Soviet Physics-JETP Letters, 6, 316-317)

[33] Rueda, A., Haisch, B. and Tung, R. (2001) Gravity and the Quantum Vacuum Inertia Hypothesis. 1. Formalized Groundwork for Extension to Gravity.

[34] Puthoff, H.E. (1989) Gravity as a Zero-Point-Fluctuation Force. https://doi.org/10.1103/PhysRevA.39.2333

[35] Ridgely, C.T. (2001) Gravitation and Forces Induced by Zero-Point Phenomena.

[36] Coughlan, G.D. and Dodd, J.E. (1991) The Quantum Vacuum. In: The Ideas of Particle Physics, Cambridge University Press, Cambridge, 33-34.

[37] Greene, B. (2005) The Fabric of the Cosmos: Space, Time and the Texture of Reality. Vintage, New York, 434-435.

[38] Straumann, N. (2002) On the Cosmological Constant Problem and the Astronomical Evidence for a Homogeneous Energy Density with Negative Pressure.

[39] Jodra, S. (2002) La face obscure de l'expansion cosmique. Science \& Vie, Hors-Série, No. 221, 130.

[40] Lachièze-Rey, M. (2004) La constante cosmologique. Pour la Science, Dossier, Dec., 98. 
[41] Rueda, A. and Haisch, B. (1998) Physics Letters A, 28, 1057. https://doi.org/10.1023/A:1018893903079

[42] Rueda, A. and Haisch, B. (1998) Physics Letters A, 240, 115-126. https://doi.org/10.1016/S0375-9601(98)00153-4

[43] Sunahata, H. (2005) Interaction of the Quantum Vacuum with an Accelerated Object and Its Contribution to Inertia Reaction Force. https://www.researchgate.net/publication/241528772

[44] Rueda, A. and Haisch, B. (1998) Foundations of Physics, 28, 9, 19.

[45] Will, C. (1989-1998) The Renaissance of General Relativity. In: The New Physics, Cambridge University Press, Cambridge, 7-33.

[46] Barry, P. (2007) Testing the Equivalence Principle [“Mr. Galileo Was Right.”], Physorg, May 21. Quotation of C. Will Saying That EP Could Fail at Subtle Level.

[47] Jaekel, M.T. and Reynaud, S. (2005) Gravity Tests in the Solar System and the Pioneer Anomaly.

[48] Gouin, R.Y. (2004) An Astrophysical Origin to Anomalous Spacecraft Accelerations. https://www.academia.edu/24130570

[49] Haisch, B. and Rueda, A. (1997) The Astrophysical Journal, 488, 563-565. https://doi.org/10.1086/304731

[50] Yam, P. (1997) American Scientific, 277, 83. https://doi.org/10.1038/scientificamerican1297-82

[51] Feynman, R. (1987) Lumière et matière. InterÉditions Points-Sciences, Paris, 195-196.

[52] de Broglie, L. (1937) Matière et Lumière. Edition Albin Michel, Paris, 109.

[53] Cluny, H. (1959) La Science Nucléaire. Buchet/Chastel, Paris, 84.

[54] Gamow, G. (1962) Gravitation. P-B Payot, Paris, 142-143.

[55] Alpher, R.A. (1962) Nature, 196, 367-368. https://doi.org/10.1038/196367b0

[56] Reynaud, S. (1997) L'énergie du Vide. Science \& Avenir, No. 112, 45.

[57] Ranada, A.F. (2004) Foundations of Physics, 34, 1955-1971.

[58] Valev, D. (2008) Journal Aerospace Research in Bulgaria, 22, 68-82. http://arxiv.org/abs/hep-ph/0507255

[59] Demaret, J. (1991) Univers. Le Mail, 138.

[60] Allais, M. (1997) L'Anisotropie de l'Espace. Edition Clément Juglar.

[61] Bagdoo, R. (2009) Link between Allais Effect and General Relativity's Residual Arc during Solar Eclipse. http://vixra.org/abs/1302.0089 https://www.academia.edu/5537473

[62] Bagdoo, R. (2012) Galaxy Rotation Curves Traced Out by the Theory of Relation. http://vixra.org/abs/1304.0079

https://www.academia.edu/5539791 